Biol. Cybernetics 17, 77-79(1975)

(C) by Springer-Verlag 1975

\title{
An Approach to the Analysis of the Underlying Structure of Visual Space Using a Generalized Notion of Visual Pattern Recognition
}

\author{
David H. Foster \\ Imperial College of Science and Technology, Applied Optics Section, \\ Department of Physics, London, Great Britain
}

Received: April 6, 1974

\begin{abstract}
An approach is described by which certain deductions can be made concerning the natural underlying structure of visual space. The procedure is indirect and makes use of the notion of visual recognition defined with respect to an arbitrarily fixed structure. Consideration of the set of mappings associated with such recognition is shown to lead to a condition that must be satisfied by any proposed underlying structure.
\end{abstract}

\section{Introduction}

The visual system establishes, via the retina, a certain representation of Euclidean three-dimensional space. In the analysis of this visual space there arise two distinct problems concerning the specification of a mathematical structure:

$\left(1^{\circ}\right)$ For a given species of structures, which particular structure of this species is assigned by the visual system to visual space?

$\left(2^{\circ}\right)$ With no constraints imposed on the species of structures from which it is drawn, what is the natural structure of visual space?

The emphasis of the present study is upon the second problem; aspects of the first have been explored by, amongst others, Luneburg (1948) and Blank $(1953,1958)$ for the species of structure of a Riemannian manifold, and Zeeman (1962) for the species of topological structures.

We put forward here an indirect approach to the analysis of the underlying structure of visual space. We make use of the general notion of visual recognition defined with respect to an arbitrarily fixed structure and obtain a condition that any candidate for the underlying structure must satisfy. Although it is not known a priori whether this condition yields a unique structure, it certainly allows the elimination of several non-trivial structures, including that of a metric space.

It should be remarked that Leibovic et al. (1971) have also examined the possibility of a connection between visual recognition and the structure of visual space. This, however, was for the case in which the latter is equipped with a certain natural polar coordinate system.

\section{The Approach}

We first fix the notation and introduce some preliminary definitions. For the sake of simplicity, we consider only the two-dimensional monocular case.

Let $S^{2}$ be a large fixed sphere centred at the eye and let $S_{*}^{2}$ be the set of those points in $S^{2}$ which map into points of visual space. Provided all sets, mappings, and the like defined on $S_{*}^{2}$ are understood to be specified only to within visual indistinguishability (Zeeman, 1962), we can treat this association as a bijection. Let $\boldsymbol{R}$ denote the reals. In conjunction with $S_{*}^{2}$, we fix a mapping $C: S_{*}^{2} \rightarrow \boldsymbol{R}$, the background field, which assigns to each point $p \in S_{*}^{2}$, unless otherwise indicated, some specified luminance $C(p) \geqq 0$. (Suppose white-light stimuli.) A visual object or pattern on $S_{*}^{2}$ is, at least, a mapping $A$ of a non-empty subset $U_{A}$ of $S_{*}^{2}$ into $\boldsymbol{R}$ such that $A(p) \geqq 0$ is the luminance of the object at the point $p \in U_{A}$. The set $U_{A}$ is referred to as the domain of the object $A$.

Let $\mathscr{S}$ be an arbitrarily fixed structure on $S_{*}^{2}$, where "structure" is to be interpreted in the general sense of Bourbaki (1968) (see Appendix). Consider, for example, the unique topological structure or the unique metric structure or the unique differentiable structure induced on $S_{*}^{2}$ by the corresponding standard structure on $S^{2}$ (though in the last case $S_{*}^{2}$ would have to open in the standard topology on $S^{2}$ ). Of course, $\mathscr{P}$ need not arise as an induced structure. For a given structure $\mathscr{P}$ on $S_{*}^{2}$, let $\mathscr{U}_{\mathscr{S}}$ be the set of all subsets $U$ of $S_{*}^{2}$ on which the induced structure can be defined, and let $\Gamma_{\mathscr{S}}$ be the set of all bijections $f: U \rightarrow U^{\prime}\left(U, U^{\prime} \in \mathscr{U}_{\mathscr{S}}\right)$ which preserve the induced 
structure, that is, if $f: U \rightarrow U^{\prime}$ is an element of $\Gamma_{\mathscr{S}}$, then $f$ is an isomorphism of the set $U$, endowed with the structure induced by $\mathscr{P}$ on $U$, onto the set $U^{\prime}$, endowed with the structure induced by $\mathscr{S}$ on $U^{\prime}$ (see Appendix). We refer to the members of $\Gamma_{\mathscr{S}}$ as local isomorphisms of $\mathscr{S}$, and write $\Gamma_{\mathscr{S}}(U)$ for the set of local isomorphisms in $\Gamma_{\mathscr{S}}$ having domain $U$. The local isomorphisms of the structures given above are the local homeomorphisms, the local isometries, and the local diffeomorphisms, respectively.

For each structure $\mathscr{S}$ on $S_{*}^{2}$, we shall consider only those objects $A$ on $S_{*}^{2}$ whose domains $U_{A}$ belong to $\mathscr{U}_{\mathscr{c}}$. An object is then to be treated as a pair: a luminance distribution and an induced structure. Given a local isomorphism $f \in \Gamma_{S \mathcal{S}}(U)$, we define its action on the set $\mathscr{F}(U)$ of all objects with domain $U$ by setting

$$
\begin{aligned}
& (f(A))(p)=A\left(f^{-1}(p)\right) \text { for all } A \in \mathscr{F}(U) \\
& \text { and } p \in f(U) .
\end{aligned}
$$

This assigns to each point $p$ in the domain of the transformed object $f(A)$ the luminance at its preimage.

We next describe the notion of visual recognition defined with respect to an arbitrarily fixed structure $\mathscr{S}$. Given objects $A$ and $B$ on $S_{*}^{2}$, visual recognition with respect to $\mathscr{S}$ of object $A$ as object $B$ is defined as the setting up, by the visual system, of a bijection $f$ of $A$ onto $B$ for some $f$ belonging to $\Gamma_{\mathscr{C}}\left(U_{A}\right)$. In the experimental situation, we infer that recognition with respect to $\mathscr{S}$ has been effected if (and only if), by visual inspection, an affirmative answer can be given to the following question: Given the structure $\mathscr{S}$ and the object $A$, is the object $B$ equal to $f(A)$ for some $f$ in $\Gamma_{\mathscr{S}}\left(U_{A}\right)$ ? For example, when $\mathscr{S}=s_{1}$, the metric structure induced on $S_{*}^{2}$ by the standard metric structure on $S^{2}$, we enquire whether object $B$ can be obtained from object $A$ by application of (the restriction of) a rotation or reflection of $S^{2}$, about or through the centre, respectively.

Trivially, a necessary condition for recognition with respect to a given structure $\mathscr{S}$ of an object $A$ as an object $B$ is that $B=f(A)$ for some $f$ in $\Gamma_{\mathscr{A}}\left(U_{A}\right)$. It is certainly not always sufficient (Dearborn, 1899; Arnoult, 1954; Foster, 1973b).

We are now ready to develop our approach to the analysis of the underlying structure of visual space, where, as we indicated in the Introduction, the underlying structure is the one with which we suppose the space is naturally endowed when there are no restrictions upon species. The possibility that this structure is the trivial structure of a set (see Appendix) is not excluded. We make the following hypothesis. There exists, built into the visual system, a certain set of mappings of visual space which preserve the underlying structure of the space; if a bijection is set up between two objects on visual space, then it is drawn from this set. (This hypothesis says, in effect, that the visual system does not carry mappings which are incompatible with the underlying structure. Note that we could have defined the underlying structure to be one of the richest structures for which the local isomorphisms include the hypothesized mappings. The present approach is preferred for heuristic reasons.) By means of the aforementioned "bijection" of $S_{*}^{2}$ onto visual space, let the structure $\mathscr{S}_{0}$ on $S_{*}^{2}$ correspond to the underlying structure on visual space and let the set $\Gamma_{\mathscr{S}_{0}}^{*}$ of local isomorphisms in $\Gamma_{\mathscr{S}_{0}}$ correspond to the hypothesized set carried by the system. Set $\Gamma_{\mathscr{S}_{0}}(U)^{*}=\Gamma_{\mathscr{S}_{0}}^{*} \cap \Gamma_{\mathscr{S}_{0}}(U)$.

Suppose an experiment is performed in which objects $A$ and $B$ on $S_{*}^{2}$ are presented to the visual system and recognition with respect to some chosen structure $\mathscr{S}$ on $S_{*}^{2}$ is asked for. Suppose, further, that this recognition is deduced to have been effected. We conclude that the visual system can set up a bijection $f$ of $A$ onto $B$ for some $f$ belonging to $\Gamma_{\mathscr{S}}\left(U_{A}\right)$. Let $\Gamma_{\mathscr{A}}\left(U_{A}\right)^{*}$ denote the subset of $\Gamma_{\mathscr{S}}\left(U_{A}\right)$ consisting of all these local isomorphisms $f$ which can be thus effected. By hypothesis,

$$
\Gamma_{\mathscr{S}}\left(U_{A}\right)^{*} \subset \Gamma_{\mathscr{S}_{0}}\left(U_{A}\right)^{*} .
$$

This inclusion will give us the desired condition for $\mathscr{S}_{0}$. By experimentation, we determine, for each subset $U$ of $S_{*}^{2}$ and for each structure $\mathscr{S}$ on $S_{*}^{2}$ that gives the induced structure on $U$, the subsets $\Gamma_{\mathscr{S}}(U)^{*}$. Let $M_{U}$ denote the union over all $\mathscr{S}$ of these sets, that is,

$$
M_{U}=\bigcup_{\mathscr{S}} \Gamma_{\mathscr{S}}(U)^{*}
$$

Since the special case $\mathscr{S}=\mathscr{S}_{0}$ is included somewhere in this union, we have $M_{U} \supset \Gamma_{\mathscr{S}_{0}}(U)^{*}$. But from (1), we also have $M_{U} \subset \Gamma_{\mathscr{S}_{0}}(U)^{*}$. Hence,

$$
M_{U}=\Gamma_{\mathscr{S}_{0}}(U)^{*} \text {. }
$$

Since $\Gamma_{\mathscr{S}_{0}}(U)^{*} \subset \Gamma_{\mathscr{S}_{0}}(U)$, any candidate $\mathscr{S}^{\prime}$ for the underlying structure $\mathscr{S}_{0}$ must thus satisfy the following condition.

(C) The set $\Gamma_{\mathscr{S}^{\prime}}$ of all local isomorphisms of $\mathscr{S}^{\prime}$ includes the sets $M_{U}$ for all $U \subset S_{*}^{2}$.

In the next section we describe a consequence of this condition.

\section{Remarks}

The approach we have advocated for the analysis of the underlying structure of visual space is essentially negative: it gives us a technique for deciding 
on some of the structures it cannot be, rather than for determining precisely which structure it is. The structures it does rule out, however, are not necessarily without significance. Consider the following example. Let $s_{1}$ and $s_{2}$ be the metric and geodesic structures on $S_{*}^{2}$ induces by the corresponding standard structures on $S^{2}$. (The standard geodesic structure on $S^{2}$ is just the set of all $V C S^{2}$ such that $V$ is a subset of a great circle of $S^{2}$.) Note that the metric and geodesic structures assigned by the visual system to visual space are isomorphic to structures on $S_{*}^{2}$ which are indistinguishable on small neighbourhoods from $s_{1}$ and $s_{2}$, respectively (see Blank, 1958). It is obvious that, locally, the set $\Gamma_{s_{2}}^{*}$ of all local isomorphisms of $s_{2}$ which can be visually effected includes elements of $\Gamma_{s_{2}}$ which are not in $\Gamma_{s_{1}}$. Condition (C) then implies that the underlying structure is not a metric structure.

With reference to the general task of determining the sets $\Gamma_{\mathscr{S}}^{*}$, it is mentioned that there exists a partial alternative to direct evaluation when there is a differentiable structure underlying the structure $\mathscr{S}$. This alternative depends upon a hypothesis connecting visual recognition and a certain visual apparentmotion effect. It is described fully in Foster (1972, 1973a).

\section{Appendix}

We summarize, informally, some basic notions in Bourbaki's theory of structures relevant to the present study (see Bourbaki, 1968).

(a) Let $E_{1}, \ldots, E_{n}$ be sets. An echelon of scheme $S$ on the base sets $E_{1}, \ldots, E_{n}$ is a set $S\left(E_{1}, \ldots, E_{n}\right)$ obtained from the sets $E_{1}, \ldots, E_{n}$ by taking their sets of subsets or products in various combinations and orders according to a scheme defined by $S$. For example, consider $S\left(E_{1}, E_{2}\right)=\mathscr{P}\left(\mathscr{P}\left(E_{1}\right) \times E_{2}\right)$, where $\mathscr{P}(E)$ denotes the set of subsets of $E$.

(b) The relation $T(E, s)$ :

$$
s \in S\left(E, A_{1}, \ldots, A_{m}\right)
$$

is called a typification of the element $s$, and a relation $R(E, s)$ is said to be transportable with respect to the typification $T$, with $E$ being considered as principal base set and the $A_{i}(1 \leqq i \leqq m)$ as auxiliary base sets, if the following condition is satisfied:

(1) $T(E, s)$ and ( $f$ is a bijection of $E$ onto $F$ )

implies

(2) $R(E, s) \Leftrightarrow R\left(F, s^{\prime}\right)$

where $s^{\prime}=\left\langle f, \mathrm{Id}_{1}, \ldots, \mathrm{Id}_{m}\right\rangle^{s}(s),\left\langle f, \mathrm{Id}_{1}, \ldots, \mathrm{Id}_{m}\right\rangle^{s}$ being the canonical extension, to the set $S\left(E, A_{1}, \ldots, A_{m}\right)$, of the bijection $f$ and the identity mappings $\operatorname{Id}_{i}$ of the $A_{i}(1 \leqq i \leqq m)$.

(c) A species of structures $\Sigma$ is formed from the following:

(1) a principal base set $E$;

(2) auxiliary base sets $A_{1}, \ldots, A_{m}$ ( $\Sigma$ need not have any auxiliary base sets);

(3) a typification $T(E, s)$ :

$$
s \in S\left(E, A_{1}, \ldots, A_{m}\right)
$$

$T(E, s)$ is called the typical characterization of the species of structures $\Sigma$;

(4) a relation $R(E, s)$ which is transportable with respect to the typification $T$, with $E$ being the principal base set and the $A_{i}$ the auxiliary base sets; $R$ is called the axiom of the species of structures $\Sigma$.

The set of elements $V$ of $S\left(E, A_{1}, \ldots, A_{m}\right)$ which satisfy the relation $R(E, V)$ is the set of structures of the species $\Sigma$ on $E$.

Consider the following two examples: first, the species of structures which has no auxiliary base sets, the typical characterization $V \in \mathscr{P}(\mathscr{P}(E))$, and as axiom the transportable relation

$$
\begin{gathered}
(\forall V)\left(\left(V^{\prime} \subset V\right) \Rightarrow\left(\left(\bigcup_{X \in Y^{\prime}} X\right) \in V\right)\right) \\
\text { and }(\forall X)(\forall Y)((X \in V \text { and } Y \in V) \Rightarrow((X \cap Y) \in V)) \text {; }
\end{gathered}
$$

this species of structures is of course the species of topological structures; second, the species of structure which has no auxiliary base sets, no typical characterization and no axiom; this species of structure is the structure of a set.

(d) Let $U, U^{\prime}$ be structures of species $\Sigma$ on sets $E, E^{\prime}$, respectively. Let $f$ be a bijection of $E$ onto $E^{\prime}$. Then $f$ is an isomorphism of the set $E$, endowed with the structure $U$, onto the set $E^{\prime}$, endowed with the structure $U^{\prime}$, if

$$
\left.\left\langle f, \mathrm{Id}_{1}, \ldots, \mathrm{Id}_{m}\right\rangle\right\rangle^{S}(U)=U^{\prime},
$$

where, as in $(b),\left\langle f, \mathrm{Id}_{1}, \ldots, \mathrm{Id}_{m}\right\rangle s$ is the canonical extension, with scheme $S$, of the bijection $f$ and the identity mappings $\operatorname{Id}_{i}$ of the $A_{i}(1 \leqq i \leqq m)$.

\section{References}

Arnoult, M.D.: Shape discrimination as a function of the angular orientation of the stimuli. J. exp. Psychol. 47, 323-328 (1954)

Blank, A.A.: The Luneburg theory of binocular visual space. J. opt. Soc. Amer. 43, 717 - 727 (1953)

Blank, A.A.: Axiomatics of binocular vision. The foundations of metric geometry in relation to space perception. J. opt. Soc. Amer. 48, 328 - 334 (1958)

Bourbaki, N.: Theory of sets. Chapter IV. Paris: Hermann, Reading, Mass.: Addison-Wesley 1968

Dearborn, G.V.N.: Recognition under objective reversal. Psychol. Rev. 6, 395 -. 406 (1899)

Foster,D.H.: A method for the investigation of those transformations under which the visual recognition of a given object is invariant: I. The theory. Kybernetik 11, 217-222 (1972)

Foster,D.H.: A hypothesis connecting visual pattern recognition and apparent motion. Kybernetik 13, 151 - 154 (1973a)

Foster, D.H.: An experimental examination of a hypothesis connecting visual pattern recognition and apparent motion. Kybernetik 14, $63-70$ (1973b)

Leibovic, K.N., Balslev,E., Mathieson,T.A.: Binocular vision and pattern recognition. Kybernetik 8, 14-23 (1971)

Luneburg, R.K.: Metric methods in binocular visual perception. In: Studies and essays. New York: Interscience Publ. Inc. 1948

Zeeman,E.C.: The topology of the brain and visual perception. In: The topology of 3-manifolds and related topics. New Jersey: Prentice-Hall 1962.

Dr. D. H. Foster

Imperial College of Science and Technology, Dept. of Physics Prince Consort Road London SW 7, 2 BZ

Great Britain 